\title{
Metabolomic based identification of clusters that reflect dietary patterns
}

\author{
H. Gibbons ${ }^{1}$, E. Carr ${ }^{1}$, G.S. Frost ${ }^{2}$, B.A. McNulty ${ }^{1}$, A.P. Nugent ${ }^{1}$, J. Walton ${ }^{3}$, A. Flynn ${ }^{3}$, \\ M.J. Gibney ${ }^{1}$ and L. Brennan ${ }^{1}$ \\ ${ }^{1}$ School of Agriculture and Food Science, Institute of Food and Health, University College Dublin, Dublin, Republic of \\ Ireland, ${ }^{2}$ Nutrition and Dietetic Research Group, Imperial College London, London, UK and ${ }^{3}$ School of Food and \\ Nutritional Sciences, University College Cork, Cork, Republic of Ireland
}

Traditional dietary assessment methods, including food-frequency questionnaires (FFQs) and weighed food diaries, are associated with a number of errors ${ }^{(1)}$. Such errors can produce inconsistent findings in relation to food intake and disease risk. Dietary biomarkers have been suggested as an objective measure of dietary intake and the application of metabolomic technologies offers a route to the identification of new dietary biomarkers. However, dietary biomarker discovery has predominantly focused on single foods ${ }^{(2,3)}$. A novel approach, nutritypes (metabolic profiles that reflect dietary intake) has emerged facilitating a more comprehensive relationship between nutrition and disease-risk while also addressing issues of nutrient-interactions. The aim of this study is to use metabolic profiles to define dietary intake patterns and additionally link these dietary patterns with nutrient and biochemical data.

Dietary intake data and urinary data from the Irish National Adult Nutrition Survey (NANS) (www.iuna.net) was used in this analysis $(n=600)$. Dietary intake data obtained from 4-day food diaries was reduced into 34 food groups and expressed as percentage of energy intake. Urinary data was analysed using the metabolomic technique ${ }^{1} \mathrm{H}$ nuclear magnetic resonance (NMR) spectroscopy. K-means cluster analysis was applied to the metabolomic data to identify clusters. Discriminatory metabolites responsible for cluster separation were identified using Chenomx Profiler (Version 7.5, Chenomx Inc.; Edmonton, Canada). Food group, nutrient and biochemical data were compared across the clusters using independent samples t-test in IBM SPSS Statistics 20. Heatmap analysis was used to link metabolites and food group intake. Participants from the NutriTech food intake study $(\mathrm{n}=40)$ were used to investigate the ability of this model to classify people into different dietary patterns.

Cluster analysis identified two clusters; cluster $1(\mathrm{C} 1)$, the "healthy" cluster and cluster $2(\mathrm{C} 2)$ the "unhealthy" cluster. C1 had a significantly higher mean intake $(\% \mathrm{E})$ of nutritionally desirable food groups; breakfast cereals and porridge, low fat/skimmed milks and poultry while $\mathrm{C} 2$ had had significantly higher intakes of chips and processed potatoes and red meat $(P<0 \cdot 05)$. Nutrients including carbohydrates, protein, fibre, calcium, potassium, folate and vitamin $\mathrm{C}$ were also significantly higher in $\mathrm{C} 1$, while $\mathrm{C} 2$ had significantly higher intakes of fats $(P<0.05)$. Biochemical measurements; red cell folate, serum folate and 25-hydroxyvitamin D were also significantly higher in $\mathrm{C} 1$ compared to $\mathrm{C} 2(P<0 \cdot 05)$. Heatmap analysis revealed that metabolites found at higher concentrations in $\mathrm{C} 1$ and in $\mathrm{C} 2$ were positively correlated with the food groups consumed at higher percentages in $\mathrm{C} 1$ and $\mathrm{C} 2$ respectively. Validation of this model in an independent group revealed that $95 \%$ of subjects were placed into the correct dietary pattern.

The current analysis identified two distinct clusters that were reflective of a healthy and unhealthy dietary pattern intake and this was further validated using an external cohort. Future applications of this approach could be developed for rapid assignment of subjects to a dietary pattern.

This analysis was supported by NutriTech (Project Number 289511).

1. Kipnis V, Midthune D, Freedman L et al. (2002) Public Health Nutr 5(6a), 915-923.

2. Heinzmann SS, Brown IJ, Chan Q et al. (2010) Am J Clin Nutr 92, 436-443.

3. Edmands WMB, Beckonert OP, Stella C et al. (2011) J Proteome Res 10, 4513-4521. 